GU J Sci, Part C, 9(4): 645-660 (2021)

Gazi University
Journal of Science
PART C: DESIGN AND TECHNOLOGY
$\mathrm{http://dergipark.gov.tr/gujsc}$

\title{
An Overview on Reliability Analysis and Evaluation Methods Applied to Smart Grids
}

\author{
Melih BILGEN ${ }^{1}$ (D) Necmi ALTIN ${ }^{2 *}$ (D) \\ ${ }^{1}$ Gazi University, Graduate School of Natural and Applied Sciences, Department of Smart Grids, 06560,Ankara/Turkey \\ ${ }^{2}$ Gazi University, Faculty of Technology, Department of Electrical-Electronics Engineering, 06560, Ankara/Turkey
}

\section{Article Info}

Research article

Received:11.08.2021

Revision:09.11.2021

Accepted:09.112021

Keywords

Smart Grids

Reliability Analysis

Evaluation Methods

\begin{abstract}
The reliability can be defined as to carry out predefined requirements in a predefined duration. The importance of reliability analysis increases day by day as the customers become more conscious about a product or service which they buy. The application area of reliability analysis is very wide. Any product $\backslash$ system or service including smart grids can be subject of the reliability analysis. As power system technology and computer science improving, the concept of smart grids begins to take part in our lives. Therefore, it is very essential to make reliability analysis for smart grids. In this paper, reliability analysis methods applied to smart grids are focused on and the classifications in reliability analysis have been explained. Besides, distribution networks reliability concept and different methods such as simulation and analytical approaches to assess the reliability have been introduced. The applications of these methods on smart grids are well explained. In addition to these, smart grids and conventional grid is compared. Different approaches such as tree analysis, failure mode effect analysis, Markov process and Monte Carlo simulation methods are carried out with wind turbines. The strengths and weaknesses of each method were evaluated.
\end{abstract}

\section{INTRODUCTION}

In engineering, design of a product targets to satisfy minimum level of service and safety throughout its lifetime. Since design process includes many uncertainties, it is a difficult task. These uncertainties may lead to under or over design solutions. Reliability is the ability to meet specific requirements under a specified period [1] . It is an extensive term that concentrate on the capability of a product to conduct its intended function. Reliability could be described as the possibility that an item will maintain to conduct its intended function without failure for a predetermined period of time under described conditions. The product can be any item such as electronics card or a mechanic device, a mobile application, a manufacturing operation etc.

The methods that used in reliability assessment create theoretical framework in order to evaluate uncertainties in a very detailed decision plan. The main goal of reliability analysis methods is to evaluate the capability of overall design or the design's any part to preserve its safety and to continue to operate in its lifetime [2]. 
The reasons that show the importance of reliability assessment on a product can be listed as follows [3]:

$>$ Reputation: Prestige of a company is directly proportional to reliability of its product.

$>$ Customer Satisfaction: Customers are not satisfied fully if they buy reliable product. On the other hand, they are absolutely disappointed if the product which they buy is unreliable. Thus, reliability plays major role in customer pleasure.

$>$ Warranty Costs: If a device is broken while under warranty, the manufacturer has to spend money for its repair or replacement. Also, device and company may acquire bad reputation. Therefore, it is worth to note that investing on reliability analysis can save companies from unexpected cost and prevent from losing their prestige.

$>$ Repeat Business: Spending more time and effort to improve reliability of product proves that the company trust their product, care client satisfaction and ensures future business potential.

$>$ Cost Study: Companies could obtain reliability data and merge this with other cost items to indicate how their product has advantageous in terms of cost. Initially, the cost of the product or device would be increased due to additional cost of reliability analysis. However, it saves more than spent on, because of less repair and maintenance cost.

$>$ Customer Requirements: Nowadays, huge amount of clients claim that manufacturers should put efficient reliability program into action because same clients had a chance to use reliability analyzed product.

$>$ Competitive Advantage: Tremendous amount of manufacturers will announce its estimated reliability data to get acquire an advantage over its rivals who either may not announce their data or have lower data.

Nowadays, reliability analysis in smart grids is among the very popular studies [4] - [7]. Smart grids is a type of grid that is created by combining the traditional grid with communication technology which enables two-way communication between producers and consumers [8]. Transforming traditional grid into smart grid is a crucial and important step since smart grid contains modern technologies within itself. These technologies vary in wide range and they can solve a lot of problems that could not be solved in conventional grid and they can improve the performance of the subsystems of conventional grid. For example, situation awareness and the quality of the service and resiliency in case of malicious attacks can be improved with smart grids [9].

Reliability analysis is evaluated as one of the most essential attributes in smart grids [10]. Smart grid promises a well aware grid that connects every role player thanks to the implementation of modern technology into the grid. In addition to this, smart grids may have more renewable energy resources that strongly depend on weather conditions with respect to conventional grid and this leads to fluctuations on electricity generation that is unwanted by transmission system operator. These type of improvements make smart grids more valuable but also vulnerable at the same time. In order to prevent this, reliability analysis methods must be employed more frequently.

Thus, in this paper, the reliability analysis and reliability evaluation methods applied to smart grids is explained. Both simulation and analytical approaches to assess the reliability have been introduced and their applications on smart grids are discussed.

This paper is organized as follows. In Section 2, system classifications in reliability analysis is discussed in details. Section 3 explains reliability analysis approaches. Lastly, reliability evaluation methods applied to smart grids are given in Section 4.

\section{SYSTEM CLASSIFICATIONS IN RELIABILITY ANALYSIS}

Classifying principal fields in reliability analysis is quite difficult since reliability analysis involves many different topics. Thus, to gather these topics under a single roof is burdensome. A topic of a discipline 
may be seen as quite meaningless by another discipline. Therefore, a random discipline is selected to show system classification.

The concept of reliability analysis can be organized by three distinctive aspects in power systems. These are adequacy, security and quality.

$>$ The adequacy may be defined as the capability of the power systems to supply the aggregate power and energy requirement of all clients in the grid and by doing this, transmission system operator has to consider possible outage of grid equipment such as transformer, transmission line or whole power system [11].

$>$ The security can be defined as the capability of the power systems to cope unforeseen disturbances such as outage of electricity grid equipment or lightening that leads to short circuits on transmission lines [11].

$>$ The quality can be defined as the measurement of grid frequency and grid voltage [12]. It is expected that the frequency should be $50 \mathrm{~Hz}$ and the line-to-neutral voltage at distribution level should be $230 \mathrm{~V}$ in Turkey.

Reliability in power systems could be split into two perspectives namely utility perspective and customer perspective. Figure 1 depicts that the classification of the reliability for power systems [13].

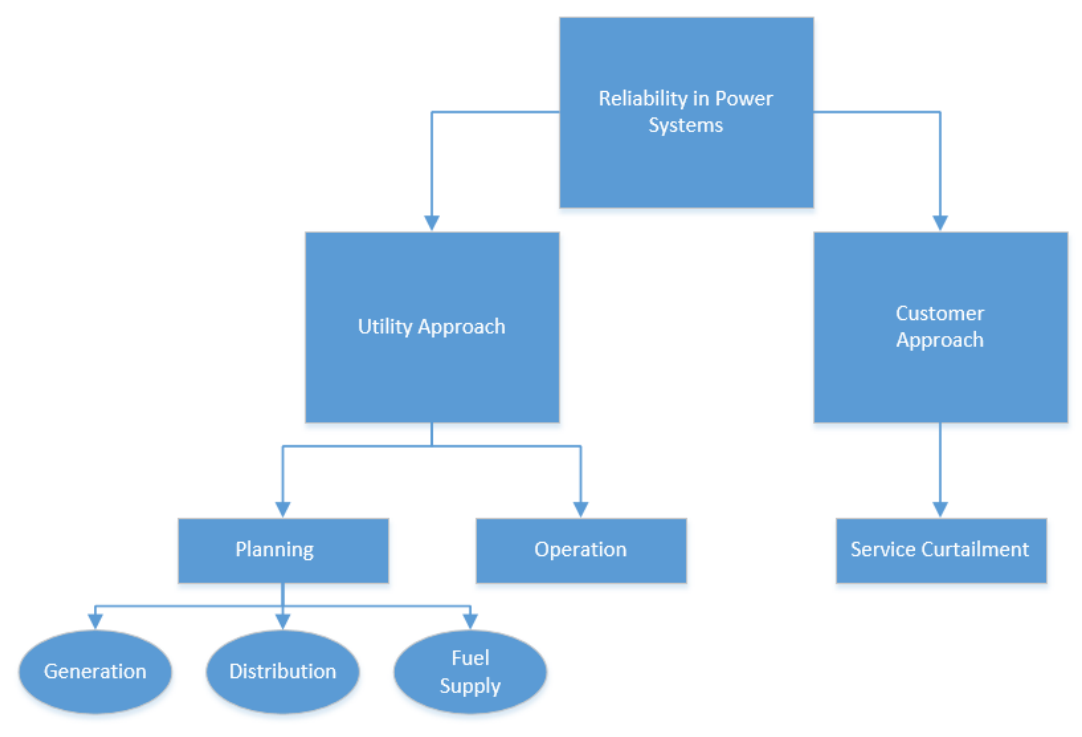

Figure 1. Perspective of on the reliability

Different perspectives can be defined by different interpretations. From customer side, the power system reliability is interested on customer end-use patterns. Indeed, what things are important for customers is related with the quality of the voltage and the grid frequency. In addition to these, another criterion for customers is duration and recurrence of electricity interruptions during a period. Thus, any interruption in electricity grid is abominable for them even if the duration of the interruption is quite short. On the other hand, utility perspective is differing from the customer perspective as specified previously. For utility perspective, the reliability is linked to both distribution side which overlaps with customer side and production side consisting of generation, transmission and distribution parts [14].

Utility perspective could be divided into two topics as the planning and operation. Additionally, the topic of planning can be sectionalized into three subtopics. These are generation, distribution and fuel supply. Generation is associated with the production of electricity and reserve margin. Reserve margin is 
described as the percentage by which the installed capacity of a country's electricity power plants outpaces the peak load. Spare capacity is essential to provide that the supply of electricity is well enough to meet demand from customer side for any condition regardless of how weather is or unforeseen an outage of $400 / 154 \mathrm{kV}$ transformer [15]. The quality of electricity distribution is related with lastingness of electricity supply. Supply continuousness is evaluated by the frequency and duration of the outages. Supply continuousness could be counted as reliability of delivery. Multiple factors can be used to assess the continuity of supply for transmission and distribution parts of the electricity grid. The last topic of planning section is the adequacy of energy supply capability. A system could be utilized to have not enough resource that will be used in electricity generation or energy supply ability as following situations exist and the forecasted energy shortage upon the demanded energy will equal or exceed 10 percent of the customer side then normal daily net energy for load, or will cause demand side to be unable to meet its energy requirements based upon use of all of the otherwise available resources and such energy deficiency will want the demand side to drop loads to end use clients [16]. Electricity grid operation from point of utility view includes quite different topics. The studies of these topics is quite time consuming because they are very technical problems and therefore reliability analyses of these are challenging. These topics are stability, security, equipment reliability, spinning and hot start reserves, zone control, harmonics, and load shedding in case of low frequency, automatic load and generation rejection.

On the other hand, customer perspective includes service curtailment. The service curtailment can also be split into many subcategories such as frequency, duration timing (day, week, month etc.), magnitude (partial or total), advance notice, persistence and coverage.

\section{RELIABILITY ANALYSIS APPROACHES}

Reliability assessment methods could be split into two main categories. These are the simulation methods and the analytical methods. Analytical approaches found on mathematical models which are used to evaluate reliability of the system factors by employing direct mathematical solutions. The primary analytical approaches are the analysis of fault tree, failure mode effect and critically analysis and the Markov model methods. On the other hand, the simulation methods could be split into the subset and the line sampling approaches. Analytical approaches are applicable for simple systems, nonetheless, it is hard to evaluate the reliability of complicated systems. A mathematical model of the system reliability is created and reliability index is determined of the load point and the overall grid in analytical approach. In addition, simulation approaches approximate the complete system-reliability factors by imitating real progress of the system and arbitrary behavior. The most commonly employed simulation approach is the Monte Carlo approach [17].

\subsection{Monte Carlo simulation approach}

The Monte Carlo simulation method could be employed in order to predict the factors by simulating the actual progress and arbitrary behavior of the grid [18]. This approach could be split into two methods. These methods are the sequential simulation and the random sampling approaches. The sequential simulation could be conducted by forming a serial of events by employing arbitrary values, and its probability distribution variables define the time for every component state. The sequential simulation approach could be split into two subclasses, too. These subclasses are the fixed interval technique and the next event technique. For both methods time is advanced. The difference between them comes from the way they handle advancing the time. In the fixed interval technique, time is advanced according to fixed steps. On the other hand, time is advanced according to occurrence of the next event in the next event technique. Principally, the random sampling approach is faster than the sequential approach and is suitable as the component failure states and repair states are autonomous [19]. Arbitrarily produced failure examples and recovery times that strongly depend on the probability distribution of the supplied statistical data are used to determine numerical outputs for reliability factors. By replicating the progress with new arbitrary numbers that are taken from input probability distributions, new probable numbers for reliability factors 
are determined. Once huge amount of iterations are made, the anticipated reliability of the system is determined, where the numbers determined for every reliability factor could be characterized via probability distribution for this factor [20].

The Monte Carlo simulation methods have also been divided into different groups by considering approaches that are employed for the sampling. Three widely used sampling approaches used in the Monte Carlo simulation can be listed as the state sampling approach, the system state transition sampling approach and the state duration sampling approach. The state sampling approach is in non-sequential form. In this approach, the status of each component is decided by considering an evenly distributed arbitrary variable that varies zero and one. In case of the arbitrary variable is bigger than the failure probability, the component is counted as up state, else it is in down state. The complete system state at every node in time is the merging of all component states. One of the major disadvantages of this approach is the approach does not take the time to repair of the system components into consideration. In the state transition sampling method, the changeover possibility from one of the state to other state is evaluated for sampling. The first step of this method is to assume that all components are in upstate in the beginning, the second step is calculation of system state duration of each component, and the third step is system state transition [21]. Lastly, the state duration sampling depends on the duration of up and down states. This approach can be evaluated as applicable to decide the duration of components states in a historical way [22]. In this method, there are two states that are success state donating as " 1 " and failure state donating as " 0 ". Figure 2 depicts two-state reliability model. Here, $\lambda$ and $\mu$ represent failure rate and repair time, respectively. The state duration distribution function of the state duration sampling approach is generally assumed as exponential [23].

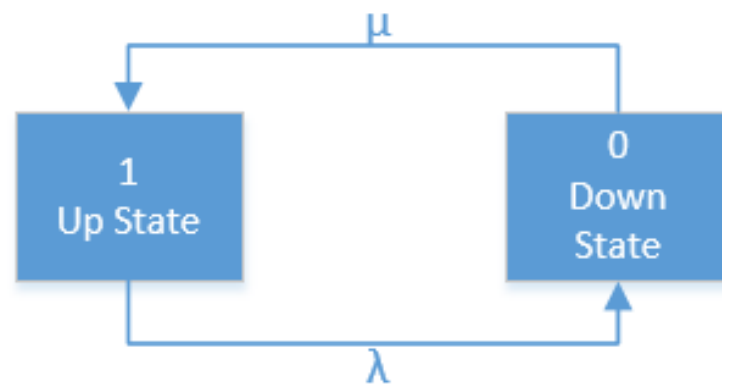

Figure 2. Two-State reliability model

\subsection{The fault tree analysis method}

The fault tree analysis method is commonly employed in risk and reliability analyses. The fault tree analysis investigates possible root causes that lead to the failure. In other words, the fault tree analysis describes actions that may lead to an unwanted failure in a grid. It reflects the connection between component failure and product [24]. Thus, by acquiring the possibilities of the causing actions, one could finish up evaluating the comprehensive possibility of the fundamental failure action [25].

Fault trees are constructed by employing gates and events. Frequently, fault trees are consisted of "AND" and "OR" gates, associating the events towards the origin of the failure. In case of a group of actions plays role in the top failure to occur, these actions are tied by employing an "OR" gate. In case of all actions would have to become true so that the main failure occurs, these all actions are tied by an "AND" gate. The reasons that leads to these actions are evaluated separately. Nonetheless, the enlargement of the tree is tied on how much exhaustive data are accessible from the past of this component [26]. The fault tree analysis method is not preferred as more precise reliability assessment is desired [27].

\subsection{The fault tree analysis method}

Failure mode and effect analysis (FMEA) could be evaluated as a progress of ranking vital parts of a device or product. By applying FMEA, one can acquire information about how a component could be failed and the repair duration of this component [28]- [29]. FMEA shows that which component or components 
are affected negatively if a component is removed deliberately. For example, all components are removed one by one of a power electronics board and other component's situations are observed. After that, a list that shows which components are critical is made. This progress has been used in very wide range of area such from power electronics boards to air planes [30].

After deciding failure stages the fundamental computation procedure includes 3 steps:

$>$ The possibilities of the failure stages occurrences have to be decided. They could be acquired from the past data for the broken parts. After that those possibilities are classified and appointed an extended value with the minimum value for the least possible division.

$>$ The rate of harshness of every failure stage is appointed and extended to the device thanks to the outcomes of the failure and destruction level.

$>$ Another extended value is appointed to the fault detection probability with the minimum value to the most expected disclosure of the failure.

\subsection{Markov process method}

A Markov process is a stochastic progress. The prospect behavior is just dependent on the current situation and it does not depend on historical data. A Markov modelling is commonly described by a cluster of discrete stages. At each stage, some probable events exist and these characterize the shifting between the present and the next stage of the progress. On the other hand, it is considered that time interval that represents each stage is exponentially distributed. In addition to this, shifting between the states are described by employing a transition rate matrix for a continuous time Markov modelling [31]. Because the grid topology is changed dynamically, it is essential to form the matrix repeatedly. Therefore, the Markov Process is fit for the grid with simple topology [13].

Markov process method could be employed in order to assess power system reliability. For component stage, a state space depiction consists of 2 stages as up and down. Up means that it is working, on the other hand down means it is not working. This simple model is defined as binary-state model, and can be broaden to comprise certain state addictiveness. Extensive models of power systems are qualified to evaluate degradation states, analysis, and distinct kinds of overhaul and fixes for a more correct depiction of the components in a real system [32].

Markov processes could be employed as Markov Decision processes (MDP) to appoint optimum determinations at distinctive stages. Markov Decision processes could be employed to model an ambiguous dynamic system where a serial of determinations have to be done over time with ambiguous results. There is an accolade related to each stage and operation done for this stage. Every operation done can either become an accolade or resulted in a cost. Therefore, in MDP, the aim is to get an optimal serial of actions. Thus, the expected accolade becomes maximum for a specified time period.

In addition to fault tree analysis, FMEA and Markov process methods, many different analytical methods employed in reliability assessment i.e. Minimum Cut-set method, Network Reduction method. Those methods consist of cutting down the number of pieces, by gathering series or parallel components in a body. The essential analytical equations consist cutting down of two pieces in series or cutting down of two pieces in parallel into single equivalent pieces as depicted in Figure 3 where $\lambda$ and $\mu$ represent the failure rate and repair time respectively [33]. 

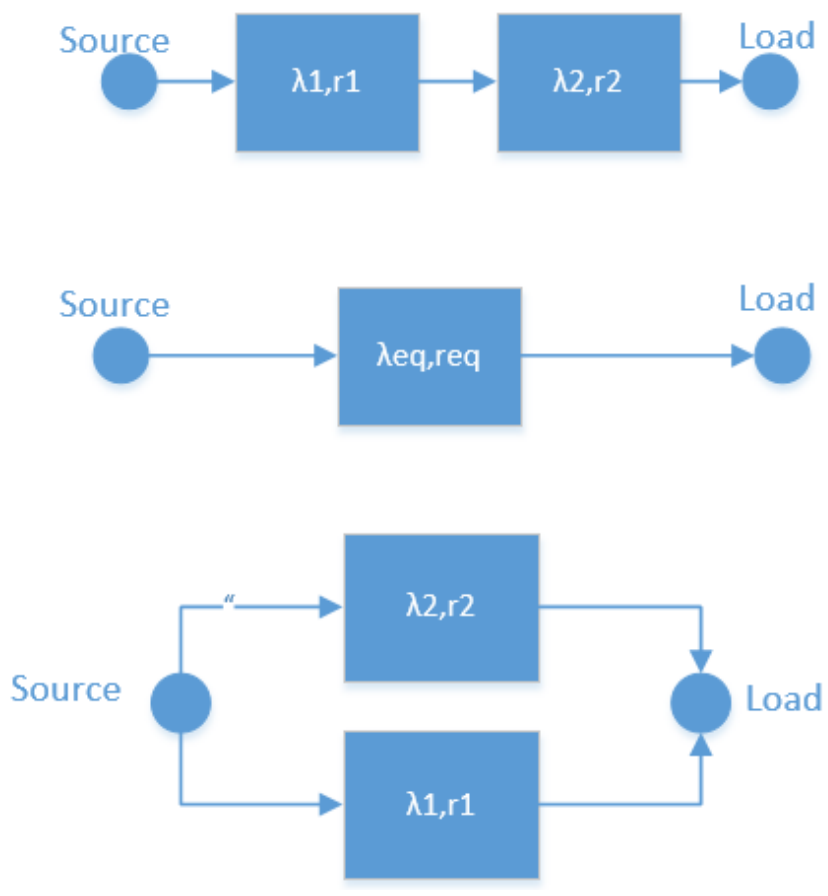

Figure 3. The equivalent system
Series

Equivalent

Parallel

For series,

$$
\begin{aligned}
& \lambda_{e q}=\lambda_{1}+\lambda_{2} \\
& \mathrm{r}_{e q}=\frac{\lambda_{1} \mathrm{r}_{1}+\lambda_{2} \mathrm{r}_{2}+\lambda_{1} \lambda_{2} \mathrm{r}_{1} \mathrm{r}_{2}}{\lambda_{e q}}
\end{aligned}
$$

For parallel,

$$
\begin{aligned}
& \lambda_{e q}=\frac{\lambda_{1} \lambda_{2}\left(\mathrm{r}_{1}+\mathrm{r}_{2}\right)}{1+\lambda_{1} \mathrm{r}_{1}+\lambda_{2} \mathrm{r}_{2}} \\
& \mathrm{r}_{e q}=\frac{\mathrm{r}_{1} \mathrm{r}_{2}}{\mathrm{r}_{1}+\mathrm{r}_{2}}
\end{aligned}
$$

The main disadvantage of an analytical method for reliability assessment is factors are mean values, and the failure and repair times are confined to be exponentially distributed.

\section{RELIABILITY EVOLUTION METHODS APPLIED TO SMART GRIDS}

Smart Grids includes wide range of features such as monitoring electricity transmission and distributions, accomplishing customer electricity management, assuring electricity transmission and distribution reliabilities and comprising two legs of at electricity distribution level. This legs are metering and engineering. One of vital topics for the electricity utilities in all around the world is the development of electricity submission reliability and presentness. The recent top performance calculation and communications technologies have had delivered new occasions for smart grids and been progressing rapid electromagnetic transient assessment permit on-line reaction to the electricity grid faults and the avoidance of interruptions [34]. The vision of the smart grids is the agenda for the advancement of the adequate, reliable and protected electricity transmission grid [35].

To develop electricity grid reliability, it is essential to establish more reliable system equipment, develop system topologies, employ remote controlled grid stations, coordinate the protection in case of 
facing over voltage and establish the equipment for rapid and reliable fault location and after fault period optimization. The number of faults is decreased considerably thanks to those precautions, and in the event of fault, they permit to decrease the time to detect the fault location, diminish the interruption costs for the clients and optimize the rebuilding of the electricity system activity.

With Smart Grids, recent technologies start to play role in electricity grid such as two-way communication. Two-way communication provides a connection between generation side and consumption side. This system could manage the client's electrical devices such as white goods to save energy, decrease electricity expense, develop quality of power. Also, this is valid for the transmission and distributions sides of the grid. By connecting distributed energy resource to smart grids the obstacles between electricity producers and consumers are eliminated thanks to flexibility and reliability [34]. Conventional electricity grid and smart grid is compared in Table 1 [36].

The main studies associated with reliability of smart grids concentrate on methods providing the reliability of the grid rather than estimating the prospect failures [37]. It makes sense when the overall topics of the reliability engineering and their precedence are evaluated. Additionally, smart grids could be accepted as a new technology if we compare it with conventional grid, and from the industrial perspective, it is logical to concentrate on improvement of methods developing the resilience and security of the smart grid.

Nonetheless, number of researchers have studies and suggested approaches for reliability assessment of smart grid. The authors of [38] suggested a method for reliability assessment of the protective systems in smart grid design. Continuous Time Markov process is employed for capturing the grid architecture and behavior specification. After that, probabilistic model checker is used to decide the possibility of these stages of the network which are evaluated as not reliable.

Table 1. Comparison of conventional grid and smart grid

\begin{tabular}{ll}
\hline Conventional Grid & Smart Grid \\
\hline Electromechanical structure & Digital structure \\
Directional communication & Bidirectional communication \\
Centralized generation & Distributed generation \\
Poor number of sensors & Sensors all around \\
Monitoring manually & Self-monitoring \\
Manual restoration & Self-healing \\
Failures and blackouts & Adaptive and island mode \\
Confined control & Common control \\
Confined options for clients & A lot of options for clients
\end{tabular}

A reliability assessment of the smart grid wireless communications system within advanced metering infrastructure (AMI) is proposed in [39]. The system has a hierarchic architecture involves home-area network (HAN), neighborhood-area network (NAN), and wide-area network (WAN). A service area is split into numerous subareas. In any sub-area, there are numerous homes where white goods are used in it. A smart meter is established in every home to predict and plan the power-expenditure of devices. The reliability is described by the availability by employing the average time between failures and average time between fixes. It explicit the possibility that the smart meter could transmit power demand to the demand management system. 
In [40], the reliability is described by the possibility of availability. Combination of the Markov chain and reliability block diagrams are employed to model the network. First one is employed in order to capture the dynamic behavior of the network and the second one captures the architecture of the network. Theoretically, their approach permits analysis of the all smart grid physical infrastructure. It is concentrated on AMI analysis. The advantage of this method is that it is less dependent on to model which has complicated interdependencies between its components.

Stochastic Petri Nets (SPN) is employed in order to assess availability and reliability of a control system networks in the smart grid in [41]. In the control center, SCADA devices and some other servers are connected with local area networks (LANs), which are preserved by the firewalls. In case of any device in this room is failed or affected by cyber-attacks, they could be fixed immediately through user interface. Furthermore, substitute servers could be employed in order to develop the reliability. Also, all control center all around the region or country are connected with secured WANs. Field employees could log into the other facilities' networks apart from control centers to repair broken devices.

Reliability and resiliency analysis method with one Static Synchronous Series Compensator is suggested and tested on a simple IEEE 9-bus test system in [42]. The model employs Markov Imbeddable Structure (MIS) model which fits for assessment of systems with the interdependent equipment. The MIS model needs description of "Functional" and "Failed" stages of the network, and calculates the network reliability as the possibility of being in one of the "Functional" stages.

A method of smart grid reliability assessment which employs the fault injection into the physical and control layer of the smart grid is suggested in [43]. A grid which includes flexible AC transmission systems (FACTS) equipment which controls the flow of the power on power lines is assessed in [43]. The authors of this paper analyze a case that a fault injected to a flow algorithm at the flexible AC transmission systems equipment spreads all over the system and leads to grid deficiency.

In [44], the reliability of smart grid is examined under different weather conditions at different locations. In this study, it is explained how varying weather conditions affects the reliability modelling and analysis in smart grids negatively. To overcome these negative effects a new approach which is called as Boolean logic driven Markov process is developed. According to this method, the reliability of subsystems such as distributed generation, system with energy storage device and solar PV plant and wind generator is assessed individually. The subsystems can be evaluated without the effect of weather, without smart grids components, with smart grid components, under normal weather influence, with the smart grid components and under stormy weather. For reliability, factors evaluated are availability and unavailability of the power supply to the specific customer - industrial, commercial, or residential. The consequences depict the development of the reliability factors with the smart grid technologies and the effect of the weather. The weather, as anticipated, affects smart grid's reliability negatively. 


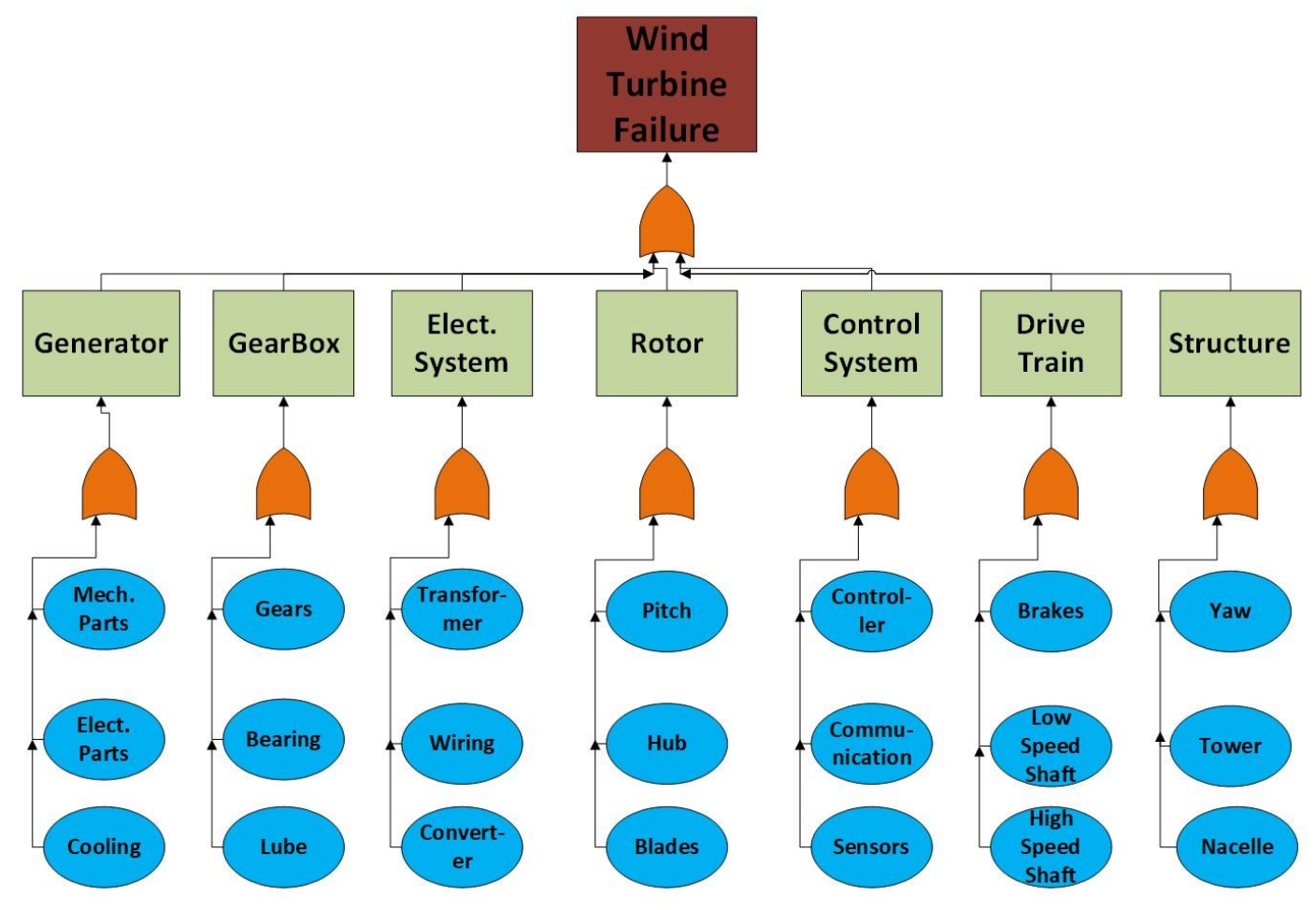

Figure 4. Standard wind turbine's fault tree

The reliability assessment analysis is made for wind turbines by employing different reliability approaches i.e. fault tree analysis, failure mode effect analysis, Markov process and Monte Carlo simulation in [45]. In this study, it is claimed that the reliability of a wind turbine could be decided by using past failure data of its parts employing Fault Tree analysis. Figure 4 depicts suggested fault tree for the wind turbine which includes reasons of main failure. The failures are so crucial that the failure of each part could prevent operation of the wind turbine. For this reason, these components are tied by "or" gates in the schema. Each of the failure leads to farther delve into to get the failure stages related to each failure. Nonetheless, the tree enlargement depends on the number of accessible past data regarding wind turbines. Wind turbines' failure data prerequisite for creating reliability model. These failure data then could be classified according to wind turbine types, so it could be get more accurate reliability model and have a chance to compare them. Another classification could be split failure data into months for the reliability analysis evaluation.

Figure 5 depicts the conventional FMEA study process of a wind turbine [46]. Nonetheless, employing FMEA on wind turbines could bring some defects. First of these defects is that particular rating scales for wind turbines have to be defined, otherwise other rating scales that belong to non-wind turbine devices have to be employed. Second defect is that huge amount of different wind turbine types and structures in the market. This makes appointing the same rating values for all of them impossible. For instance, let us assume a direct drive wind turbine's synchronous generator and a fixed speed wind turbine's induction generator are failed. The cost of repair of synchronous generator is usually more expansive than induction generator's. Also, the damage of synchronous generator is harsher. Another topic of the method is that the assessment risk priority number (RPN) could not segregate between a highly serious but low possible failure stage and a less serious with higher accruing possibility. To solve these problems, [45] suggested a modified progress for failure mode effect analysis which the prioritization of the failure stages is based on values representing the cost outcomes. 


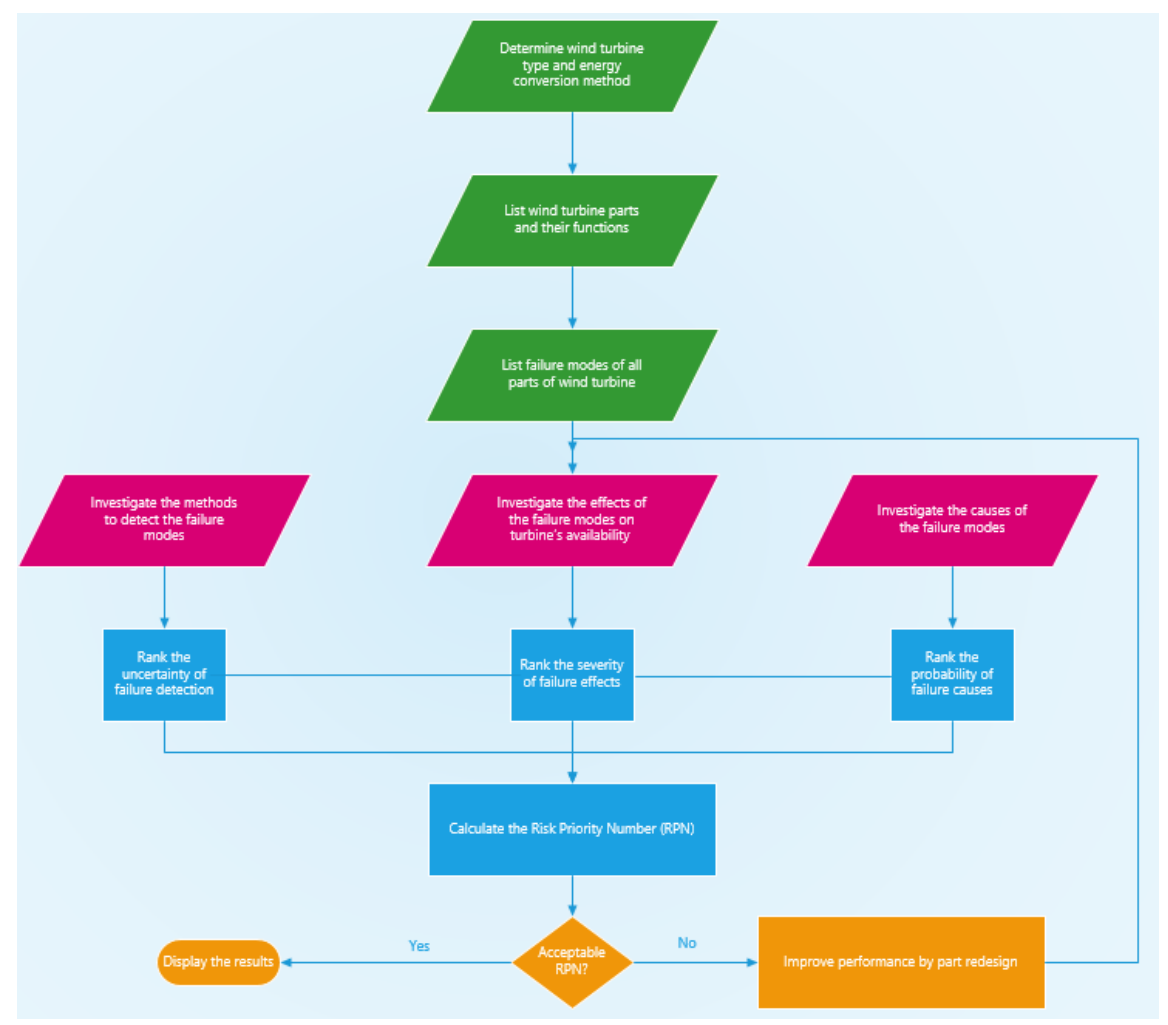

Figure 5. FMEA process for a typical wind turbine

Markov Process could be employed in wind turbine reliability in order to model the time domain operation of a set of wind turbines by paying regard to failure and fixing that wind turbines, fluctuating in wind velocity, and the load profile [45]. Calculating the reliability of a wind farm over time is useful for predicting long-term electricity generation in addition to short-term operations. It has to be done especially in the unregulated electricity market where wind farms exploit the cost advantage. 2-state Markov process is employed to present devices such as wind turbine in order to assess wind plants' reliability. These states are "working" and "failure". If this model is employed in wind plant and if the number of wind turbines in this plant is $\mathrm{k}$, then total states will be $2 \mathrm{k}$. As you guess a typical wind plant consists of multiple wind turbines, therefore the number of states are increased significantly. In this paper, a Markovian model is employed in order to work on availability and reliability of wind plant due to the fluctuations in wind velocity and load alteration for any time interval. This model is evolved by assuming that all turbines in a wind plant are same model. Wind plant modelling is made by employing Markov Chain with $(\mathrm{k}+1)$ number of states. These states symbolized the number of operating wind plants at any time. Kendall-Lee notation of this birth and death process is $\mathrm{M} / \mathrm{M} / \mathrm{S} / \mathrm{GD} / \mathrm{N} / \mathrm{N}$. M/M are used to indicate failure and repair. " $\mathrm{S}$ " is used to indicate the number of parallel repair crew. "GD" is used to indicate failed turbines waiting times are based on general queue discipline. The first of "N"s indicates that if wind turbines are failed, fixing progress has adequate capacity. Lastly, the second of "N"s indicates the number of similar wind turbines established in the wind plant. Figure 6 depicts the schema of this modeling. Here, $\lambda$ represents failure and $\mu$ represents are fixing rates. In order to clarify the fixing rates, by assuming that $k$ number of failed wind turbines was fixed together. Thus, the fixing rate is $k \times \mu$. 


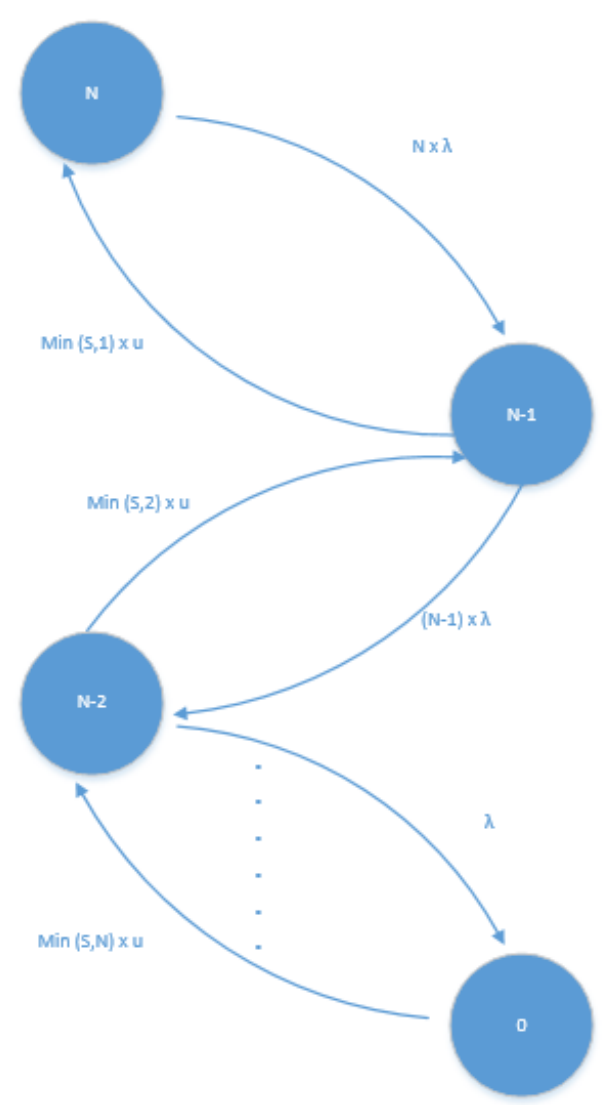

Figure 6. Rate diagram for wind plant Markov model

In [45], it is discussed that, as well as analytical methods, Monte Carlo simulation method could be employed in wind turbine reliability analysis. Figure 7 depicts the diagram of a single wind turbine model which is employed to predict output power by considering wind velocity probability distribution. The advantage of the method is independency form the wind velocity or wind turbine type. No matter how wind velocity is and what type of wind turbine is, the system could be modelled via computer program by employing block diagrams. Actually, for any time period, a wind velocity is produced by changing the position of the block in the diagram. The decision block conducts according to the definitive power curve of the wind plant to decide the amount of power that will be produced by depending on wind velocity. The simulation is conducted for several iterations and the mean anticipated power produced by the plant is computed as well as its confidence span. The anticipated mean wind energy is computed by multiplying the anticipated produced power by the number of wind turbines. 


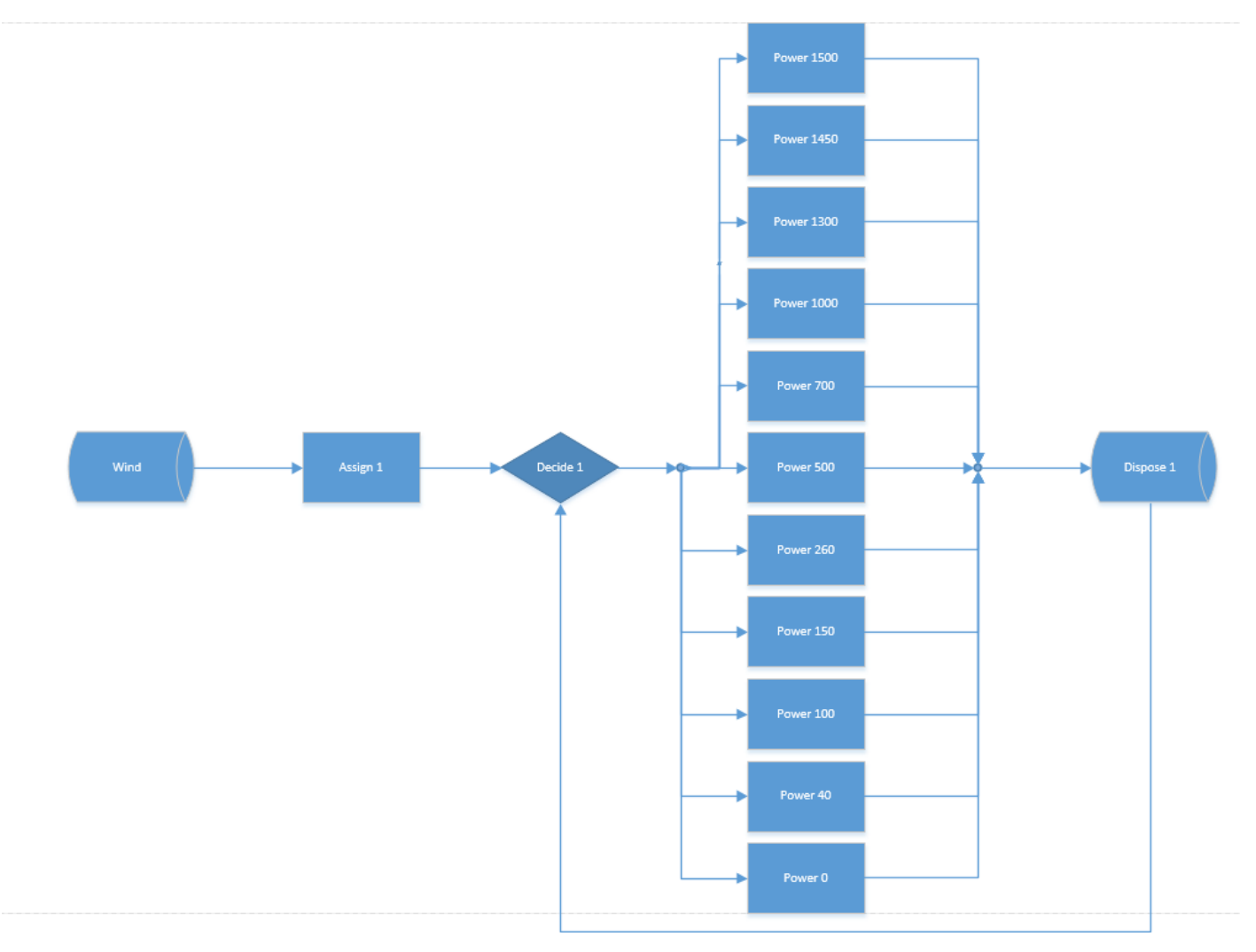

Figure 7. Wind turbine model for output power estimation

\section{CONCLUSIONS}

In this study, reliability analysis, system classifications in reliability analysis and evaluation methods applied to the smart grids are introduced. Since system classification in reliability analysis for each discipline can be very different from each other, the classification is made by considering the power system. The basic concept of distribution grids reliability and different simulation and analytical methods to assess the reliability have been explained. Analytical approaches often determine the system topology and input values, and then create a mathematical model of a system to evaluate the reliability. Simulation approaches such as Monte Carlo can determine the indices by simulating the probabilistic and arbitrary behavior of the systems. Therefore, the problem is treated as a series of experiments instead of considering the analytical models of the systems. The main weakness of simulation methods is that to obtain an acceptable level of the accuracy of the performance indices a huge amount of experiments need to run. Due to the long computational time, simulation approaches are not as popular as analytical approaches. Managing the smart grids are less difficult since the reliability of smart grids are higher and also they are more transparent with respect to conventional grid. The smart grid permits combining different electricity generation plants and guarantee reliable electric power supply for clients. Several reliability analysis methods are given for wide range of smart grid applications such as protective systems in smart grid infrastructure, smart grid wireless communications system to support demand side management within advanced metering infrastructure and control system networks in the smart grid. Also, a new method called Boolean logic driven Markov process is introduced. This method can be used for the situations like varying conditions in order to assess reliability analysis in smart grids. Four different approaches namely fault tree analysis, failure mode effect analysis, Markov process and Monte Carlo simulation methods are exemplified on wind turbines to improve the understanding of readers. 


\section{REFERENCES}

[1] A. Avizienis, J. Laprie, B. Randell and C. Landwehr, "Basic concepts and taxonomy of dependable and secure computing," IEEE Transactions on Dependable and Secure Computing, vol. 1, pp. 11-33, 2004.

[2] E. Arteaga and A. Soubra, "Reliability Analysis Methods," University of Nantes-GeM Laboratory, Nantes, 2014.

[3] "Weibull," [Online]. Available: https://www.weibull.com/basics/reliability.htm. [Accessed 5 $72020]$.

[4] J. Huan, Y. Xiao, X. Zhang, H. Liu, X. He and W. Lu, "Integrated Reliability Analysis of Power Distribution Network and Urban Road Network," in China International Conference on Electricity Distribution (CICED), Shanghai, 2021.

[5] S. Hajeforosh, Z. Nazir and M. Bollen, "Reliability Aspects of Battery Energy Storage in the Power Grid," in IEEE PES Innovative Smart Grid Technologies Europe (ISGT-Europe), Hague, 2020.

[6] S. S. Reka and T. Dragicevic, "Future effectual role of energy delivery: A comprehensive review of internet of things and smart grid," Renewable and Sustainable Energy Reviews, vol. 91, pp. 90-108, 2018.

[7] K. Moslehi and R. Kumar, "A Reliability Perspective of the Smart Grid," IEEE Transactions on Smart Grid, vol. 1, no. 1, pp. 57-64, 2010.

[8] P. Hines, V. Jason and B. Tivnan, "Smart Grid: Reliability, Security, and Reslieincy," University of Vermont, 2014.

[9] "Glossary of Terms Used in NERC Reliability Standards," NERC, 2020.

[10] E. Fuchs and M. Masoum, Power Quality in Power Systems and Electrical Machines, Academic Press, 2015.

[11] A. Heidari, "Reliability Analysis of Power Distribution System in Presence of Distributed Generation Units," The University of New South Wales, Sydney, 2015.

[12] A. Sanghvi, Cost-benefit analysis of power system reliability: Determination of interruption costs, Academic Press, 1990.

[13] "Estimating the Economically Optimal Planning Reserve Margin," El Paso Electric Co., 2015.

[14] "Hearings Before the Subcommittee on Energy Regulation of the Committee on Energy and Natural Resources, United States Senate, Ninety-sixth Congress," Committee on Energy and Natural Resources. Subcommittee on Energy Regulation, 1979.

[15] M. Wadi and M. Baysal, "Reliability Evaluation in Smart Grids via Modified Monte Carlo Simulation Method," in ICRERA, Paris, 2018.

[16] R. Billinton and L. Gan, "Use of Monte Carlo simulation in teaching generating capacity adequacy assessment," IEEE Transactions on Power Systems, vol. 6, no. 4, pp. 1571 - 1577, 1991.

[17] C. Singh and J. Mitra, "Monte Carlo Simulation for Reliability Analysis of Emergency and Standby Power Systems," in IEEE Industry Applications Conference, Thirtieth IAS Annual Meeting, Orlando, 1995. 
[18] "IEEE Guide for Electric Power Distribution Reliability Indices," IEEE Std 1366-2012 (Revision of IEEE Std 1366-2003), pp. 1-43, 2012.

[19] A. M. Giacomoni, S. Y. Goldsmith, S. M. Amin and B. F. Wollenberg, "Analysis, modeling, and simulation of autonomous microgrids with a high penetration of renewables," in IEEE Power and Energy Society General Meeting, San diego, 2012.

[20] R. Billinton and W. Li, Reliability assessment of electric power system using Monte Carlo methods, New York: Plenum Press, 1994.

[21] Y. Hegazy, M. Salama and A. Chikhani, "Adequacy assessment of distributed generation systems using monte carlo simulation," IEEE Transactions on Power Systems, vol. 18, no. 1, pp. 48-52, 2003.

[22] W. S. Lee, D. L. Grosh, F. A. Tillman and C. H. Lie, "Fault tree analysis, methods, and applications-A Review," IEEE Transactions on Reliability, Vols. R-34, no. 3, pp. 194 - 203, 1985.

[23] M. Stamatelatos, "Fault Tree Handbook with Aerospace Applications," NASA, Washington DC, 2002.

[24] W. Vesely, F. Goldberg, N. Roberts and D. Haasl, "Fault Tree Handbook," U.S. Government Printing, Washington DC, 1981.

[25] W. Li, Risk Assessment of Power Systems: Models, Methods, and Applications, Wiley-IEEE Press, 2004.

[26] S. Lee, "Probabilistic Reliability Assessment for transmission planning and operation including cascading outages," in IEEE/PES Power Systems Conference and Exposition, Seattle, 2009.

[27] C. W. Williams, "Weather Normalization of Power System Reliability Indices," in IEEE Power Engineering Society General Meeting, Tampa, 2007.

[28] D. Stamatis, "Failure Mode and Effect Analysis FMEA from Theory to Execution," ASQ, Milwaukee, 2003.

[29] O. Ibe, Markov Processes for Stochastic Modeling, Massachusetts: Academic Press, 2009.

[30] R. Billinton and R. Allan, " Reliability Assessment of Large Electric Power Systems," Kluwer Academic Publishers, 1988.

[31] "Reliability of Electric Utility Distribution Systems: EPRI White Paper," EPRI, Palo Alto, 2000 .

[32] S. Gudzius, S. Gecys, L. Markevicius, R. Miliune and M. A, "The Model of Smart Grid Reliability Evaluation," ELECTRONICS AND ELECTRICAL ENGINEERING, Kaunas, 2011.

[33] M. Hinchey and S. A. Bohner, "Innovations in Systems and Software Engineering," Springer, no. 11334, pp. 19-23, 2011.

[34] H. Farhangi, "The Path of the SmartGrid," IEEE Power and Energy Magazine, vol. 8, no. 1, pp. 18-28, 2010.

[35] S. Chren, "Multi-layered Reliability Analysis in Smart Grids," Masarykova univerzita Fakulta informatiky, Brno, 2017.

[36] A. Mahmood, O. Hasan, H. R. Gillani and Y. Saleem, "Formal reliability analysis of protective systems in smart grids," IEEE, p. 198-202, 2016. 
[37] D. Niyato, P. Wang and E. Hossain, "Reliability analysis and redundancy design of smart grid wireless communications system for demand side management," IEEE Wireless Communications, vol. 19, no. 3, pp. 38-46, 2012.

[38] J. Wäfler and P. Heegaard, "A combined structural and dynamic modelling approach for dependability analysis in smart grid," In Proceedings of the 28th Annual ACM Symposium on Applied Computing, ACM, pp. 660-665, 2013.

[39] R. Zeng, Y. Jiang, C. Lin and X. Shen, "Dependability analysis of control center networks in smart grid using stochastic petri nets," IEEE Transactions on Parallel and Distributed Systems, vol. 23, no. 9, pp. 1721-1730, 2012.

[40] M. Albasrawi, N. Jarus, K. Joshi and S. S. Sarvestani, "Analysis of reliability and resilience for smart grids," in 2014 IEEE 38th Annual Computer Software and Applications Conference, Vasteras, 2014.

[41] A. Faza, S. Sedigh and B. McMillin, "Integrated cyber-physical fault injection for reliability analysis of the smart grid," in In International Conference on Computer Safety, Reliability, and Security, Vienna, 2010.

[42] A. Islam, A. Domijan and A. Damnjanovic, "Assessment of the Reliability of a Dynamic Smart Grids System," International Journal of Power and Energy Systems, vol. 31, no. 4, 2011.

[43] S. Kahrobaee, "RELIABILITY MODELING AND EVALUATION OF DISTRIBUTED ENERGY RESOURCES AND SMART POWER DISTRIBUTION SYSTEMS," The Graduate College at the University of Nebraska, Nebraska, 2014.

[44] R. Mikulak, R. McDermott and M. Beauregard, The Basics of FMEA, CRC Press, 2008.

[45] O. Akinpelumi and K. Kopsidas, "Impact Analysis of Cyber-related Failures on Power System Reliability - A Review," in IEEE Madrid PowerTech, Madrid, 2021.

[46] Y. Dai, Z. Chen, X. Zheng, X. Dong, Y. Du and X. Liu, "Smart Electricity Meter Reliability Analysis Based on In-service Data," in 4th International Conference on Energy, Electrical and Power Engineering (CEEPE), Chongqing, 2021. 